\title{
Scientific Visualization of Magnetic Reconnection Simulation Data by the CAVE Virtual Reality System
}

\author{
Hiroaki OHTANI ${ }^{1,2)}$ and Ritoku HORIUCHI ${ }^{1,2)}$ \\ ${ }^{1)}$ National Institute for Fusion Science, Toki 509-5292, Japan \\ ${ }^{2)}$ The Graduate University for Advanced Studies (SOKENDAI), Toki 509-5292, Japan
}

(Received 23 July 2008 / Accepted 11 August 2008)

\begin{abstract}
In this study, scientific visualization by the CAVE virtual reality (VR) system is applied to the analysis of magnetic reconnection simulation data for the first time. The magnetic field streamline, the isosurface of density profile, the contour of temperature profile, the arrow of flow velocity, and other variables are displayed in VR space. The trajectories of particles under the electromagnetic field obtained by simulation are also traced simultaneously. We can clearly see the relationship between complex three-dimensional structures of magnetic fields, distribution of particle temperature, particle trajectories, and other variables in VR space.
\end{abstract}

(C) 2008 The Japan Society of Plasma Science and Nuclear Fusion Research

Keywords: particle trajectory, magnetic reconnection, CAVE, visualization

DOI: $10.1585 /$ pfr.3.054

Magnetic reconnection is widely considered to play an important role in energetically active phenomena in high temperature plasmas. In spite of intensive research, many basic questions about the details of mechanisms of reconnection still remain poorly understood. Ions become unmagnetized and execute a complex thermal motion called meandering in the ion dissipation region. The complex meandering motion leads to the growth of off-diagonal components of the pressure tensor term, which is one of main causes to break ion frozen-in condition in the vicinity of magnetic neutral sheet [1-3]. It is important to clarify the relationship between the role of meandering particles and physical quantities such as temperature and magnetic field structure in order to understand the magnetic reconnection phenomenon.

In the conventional visualization analysis, we use so called "visualization software" on graphic workstations. Because the complex particle orbits of meandering motion and three-dimensional (3-D) structure of vector fields are shown on a two-dimensional plane through the workstation monitor, it is difficult to grasp the spatial structure of the orbits and 3-D vector fields. In order to understand the role of the complex orbits of meandering particles, it is indispensable to analyze them in 3-D space by scientific visualization technology. We believe that one of the answers is virtual reality (VR).

For visualizing reconnection simulation data, a deep immersion into the VR world is needed. One of the most successful immersive VR systems is CAVE (Cave Automatic Virtual Environment) [4]. The CAVE system can produce three important views: stereo view, immersive view, and interactive view. For scientific VR visualization

author'se-mail: ohtani@dss.nifs.ac.jp using the CAVE system, general purpose VR visualization software "VFIVE" has been developed [5-8]. This software can show vector fields as lines or arrows, and scalar fields as isosurface, contour, and volume rendering. It can also trace particle motion following the flow field of simulation data; however, it cannot deal with particle motion in the electromagnetic field of simulation data. In this study, we improve VFIVE to trace the trajectories of plasma particles in the electromagnetic field obtained in the particle simulation to analyze the reconnection phenomenon in the VR world.

The equation of motion for a single particle is given by the Newton-Lorentz equation. Using the leap-frog method, we integrate six differential equations with respect to time. The electromagnetic field on the grid points is obtained from simulation data, and the force acting on the particles is calculated by the third-order interpolation of the fields at the nearest grid points. The initial velocity is given by the Box-Muller method [9] with the same average velocity as the flow velocity, which is given by simulation data. Simulation data of a collisionless driven reconnection is obtained by the 3-D PArticle Simulation code for Magnetic reconnection in an Open system (PASMO) [10-12]. The initial condition is given by a one-dimensional Harris-type equilibrium, in which the magnetic field is parallel to the $x$-axis and a function of the $y$-coordinate. At the upstream boundary ( $y$ boundary), ions and electrons enter the system by $\boldsymbol{E} \times \boldsymbol{B}$ drift due to a driving electric field $E_{z 0}$, whereas at the downstream boundary ( $x$ boundary), particles exit and enter the system under the free boundary condition.

Figure 1 shows a snapshot of the magnetic structure and density profile of magnetic reconnection data at $t \omega_{\text {ce }}=331$ in the CAVE visualization. Magnetic field 


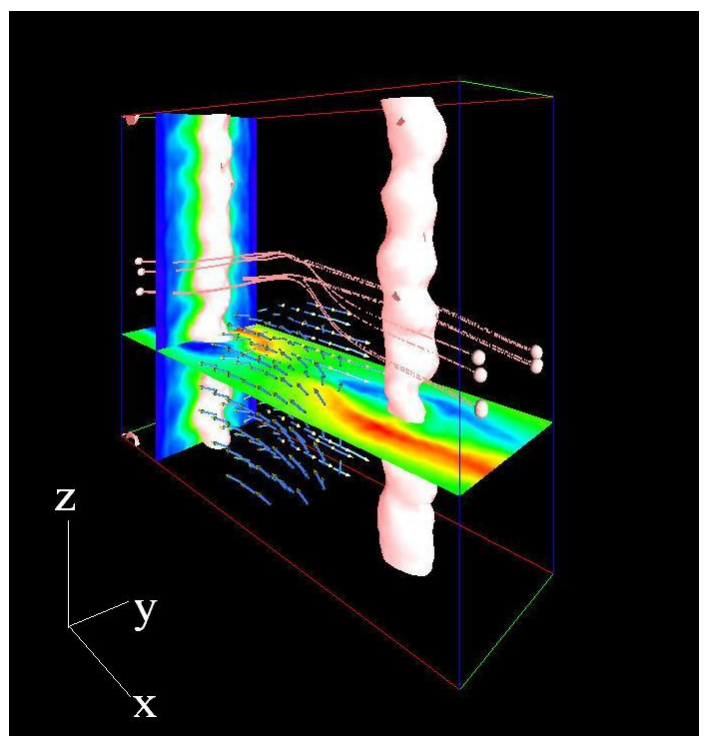

Fig. 1 CAVE visualization of magnetic structure (lines, arrows, and color contours) and density profile (isosurface and color contours).

lines are shown by pink solid lines and blue arrows, and the $z$ component of the magnetic field is mapped on the color contour in the $x y$ plane. The quadrupole structure is clearly demonstrated. The density profile is displayed by the isosurface and color contours in the $y z$ plane. These high-density regions correspond to magnetic islands approaching the downstream regions. They become unstable against a kink instability [13,14].

The top of Fig. 2 displays magnetic structure (blue lines and color contours in the $y z$ plane), ion temperature (color contours in the $x y$ plane), and trajectories of ions (white lines) near the reconnection region at $t \omega_{\mathrm{ce}}=1312$. The color contours of the reconnection component of the magnetic field $B_{x}^{2}+B_{y}^{2}$ show that a low-frequency electromagnetic instability is excited near the central region $[13,14]$. Ions coming from upstream execute a meandering motion around the magnetic neutral sheet. The amplitude of the meandering motion corresponds to the width of the high-temperature region. The bottom of Fig. 2 shows ion flow velocity (arrows) in addition to the same features as the top of Fig. 2 without the color contours of $B_{x}^{2}+B_{y}^{2}$. The arrow length corresponds to the magnitude of the flow velocity. From the distribution of arrow lengths, it is found that flow velocity is higher in the reconnection and downstream regions. Ions enter the system from the upstream boundary and exit through the downstream boundary.

Scientific visualization by VR in the CAVE system is a powerful tool for analysis of magnetic reconnection simulation data. The use of the CAVE system at the National Institute for Fusion Science is available for visiting scientists. Dr. Ohno and Dr. Kageyama at the Japan Agency for Marine-Earth Science and Technology (JAMSTEC) have contributed to the improvement of VFIVE.

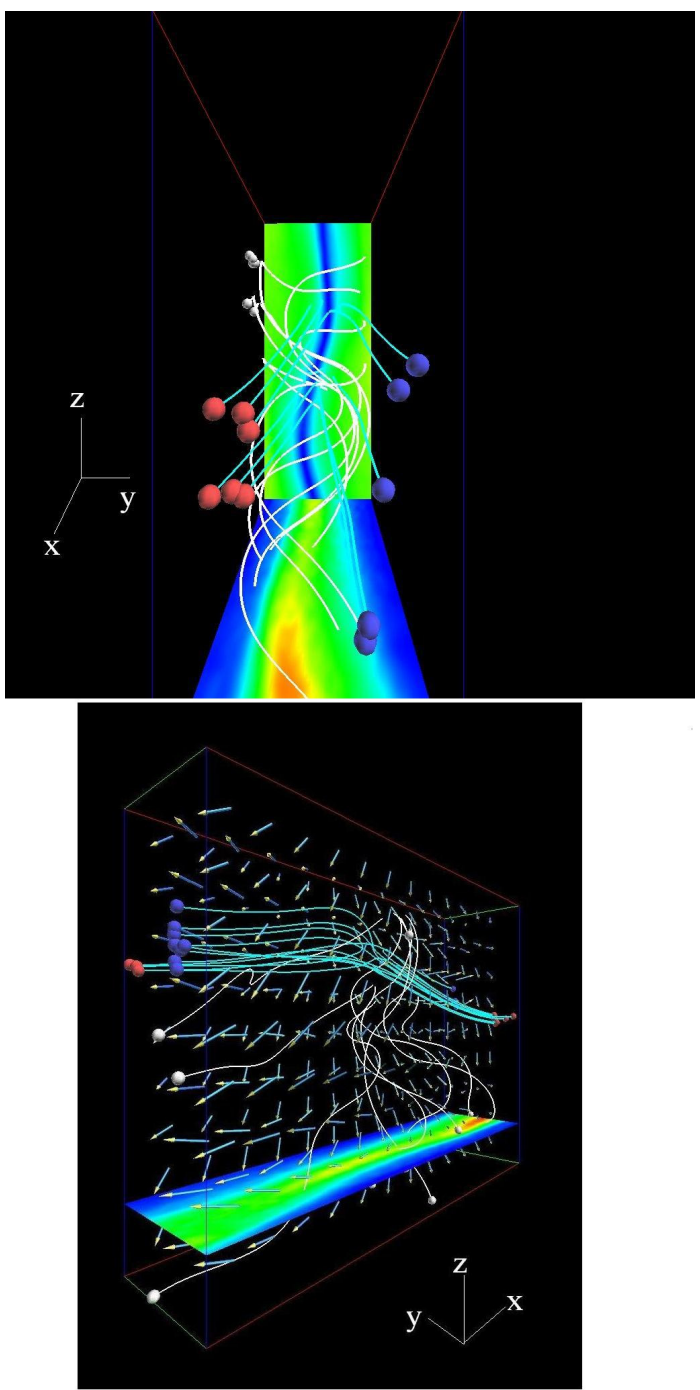

Fig. 2 CAVE visualization of (top) magnetic structure (blue lines and color contours), ion temperature profile (color contours) and trajectories of ions (white lines); and (bottom) magnetic structure, ion temperature profile, ion flow velocity (arrows), and trajectories of ions.

[1] W. Horton and T. Tajima, J. Geophys. Res. 96, 15811 (1991).

[2] A. Ishizawa and R. Horiuchi, Phys. Rev. Lett. 95, 045003 (2005).

[3] R. Horiuchi and H. Ohtani, Comm. Comp. Phys. 4, 496 (2008).

[4] C. Cruz-Neira, D.J. Sandin and T.A. DeFanti, ACM SIGGRAPH 93, 65 (1988).

[5] A. Kageyama, Y. Tamura and T. Sato, Trans. Virtual Reality Soc. Japan 4, 717 (1999).

[6] A. Kageyama, Y. Tamura and T. Sato, Progress Theor. Phys. Suppl. 138, 665 (2000).

[7] N. Ohno and A. Kageyama, J. Plasma Phys. 72, 1069 (2006).

[8] N. Ohno and A. Kageyama, Phys. Earth Planet. Interiors 163, 305 (2007).

[9] W.H. Press, S.A. Teukolsky, W.T. Vetterling and B.P. Flannery, Numerical Recipes in FORTRAN, second ed. (Cambridge University Press, Cambridge, 1992.)

[10] R. Horiuchi and T. Sato, Phys. Plasmas 6, 4565 (1999). 
[11] H. Ohtani, S. Ishiguro, R. Horiuchi, Y. Hayashi and N. Horiuchi, Lecture Notes Comput. Sci. 4759, 329 (2008).

[12] H. Ohtani and R. Horiuchi, submitted to J. Comput. Phys., (2008).
[13] R. Horiuchi, H. Ohtani and A. Ishizawa, Comp. Phys. Comm. 164, 17 (2004).

[14] H. Ohtani, R. Horiuchi and A. Ishizawa, J. Plasma Phys. 72, 929 (2006). 